\title{
Coupling in reflector arrays
}

\section{Appel-Hansen, Jørgen}

\section{Published in:}

I E E E Transactions on Antennas and Propagation

Publication date:

1968

\section{Document Version}

Publisher's PDF, also known as Version of record

Link back to DTU Orbit

Citation (APA):

Appel-Hansen, J. (1968). Coupling in reflector arrays. I E E E Transactions on Antennas and Propagation, 16(6), 759-760.

\section{General rights}

Copyright and moral rights for the publications made accessible in the public portal are retained by the authors and/or other copyright owners and it is a condition of accessing publications that users recognise and abide by the legal requirements associated with these rights.

- Users may download and print one copy of any publication from the public portal for the purpose of private study or research.

- You may not further distribute the material or use it for any profit-making activity or commercial gain

- You may freely distribute the URL identifying the publication in the public portal

If you believe that this document breaches copyright please contact us providing details, and we will remove access to the work immediately and investigate your claim. 


\section{REFERENCES}

[1] R. H. Du Hamel, "Pattern synthesis for antenna arrays on circular, elliptical, and spherical surfaces," Electrical Engineering Research Lab., Lniversity of Illinois, Urbana, Ill., Tech. Rept. 16, May 1952.

[2] C. E. Hickman, H. P. Neff, and J. D. Tillman, "The theory of single-ring circular antenna arrays." Engineering Experiment Station, University of Tennessee, Knorville, Sci. Rept. 4, July 1961.

[3] C. O. Stearn and A. C. Stewart, "An investigation of concentric ring antennas with low sidelobes," IEEE Trans. Antennas and Propagation, vol. AP.13, pp. 856-863, November 1965.

[4] M. T. Ma and L. C. Walters, "Synthesis of concentric ring antenna arrays yielding approximately equal sidelobes," Radio Sci., vol. 3 (New Series), pp. 465-470, May 1968 .

[5] D. K. Cheng and F. I. Tseng. "Maximisation of directive gain for circular and elliptical arrays," Proc. IEE (London), vol. 114, pp. 589-594. May 1967.

[6] H. Mott and J. E. Dudgeon, "On circular array pattern synthesis using a digital computer," Proc. IEEE (Letters), vol. 56, pp. 369-370, March 1968.

\section{Coupling in Reflector Arrays}

\section{INTRODUCTION}

For many applications, such as echo enhancement and satellite communication, reflectors which have a maximum of reradiation back in the direction of arrival of an incident wave are needed. In 1955, Van Atta [1] proposed a reflector with this property. The reflector consists of antenna elements properly arranged and connected by transmission lines. In order to reduce the space occupied by a reflector array, it is desirable to arrange the array antennas as close to each other as possible. However, in this case coupling between the array antennas will reduce the reflecting properties of the reflector array.

The purpose of the present communication is to demonstrate that this is true for a Van Atta reflector consisting of four halfwave dipoles arranged on a line. Some theoretical studies of the influence of coupling in Van Atta reflector arrays have been carried out previously [2]-[4]. In this communication, theoretical results obtained by using the methods described in [2]-[4] will be compared with experimental results. In contrast to previous experimental results [4] which have been presented as normalized, the results described here are absolute. Before the experimental results are described, some theoretical remarks will be given.

\section{THEORY}

For a linear Van Atta reflector consisting of $n$ parallel half-wave dipoles, we will determine the maximum back-scattering cross section for an incident wave polarized parallel to the dipoles and propagating along the normal to the plane containing the dipoles. This back-scattering cross section will be determined when coupling is neglected. Then there are two fields which add up to give the cross section. First, the field due to scattering from the dipoles when they receive the incident wave; second, the field due to the interconnections between the dipoles [4]. If the length of the transmission lines is chosen to be an unequal number of half a wavelength, these two fields add in phase and we have the maximum cross section for normal incidence. It is well-known that for one dipole, the backscattering cross section of the field due to scattering is $0.20 \lambda^{2}[5]$. Since the field intensity due to the interconnecting transmission lines is of the same magnitude as the field intensity due to scattering, the maximum back-scattering cross section of one dipole is $4 \times 0.20 \lambda^{2}$, i.e., the maximum back-scattering cross section for normal incidence is $0.80 n^{2} \lambda^{2}$ for the
Van Atta reflector. This result shows that if a certain space is available, the cross section increases with the square of the number of dipoles arranged within the space. However, the cross section cannot be increased indefinitely, because coupling will reduce the backscattering cross section when the dipoles are

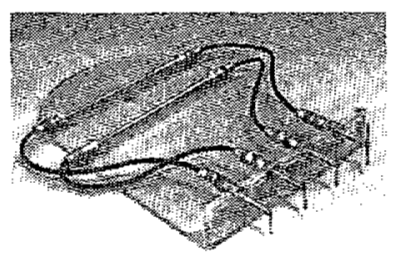

Fig, 1. The experimental Van Atta reflector.

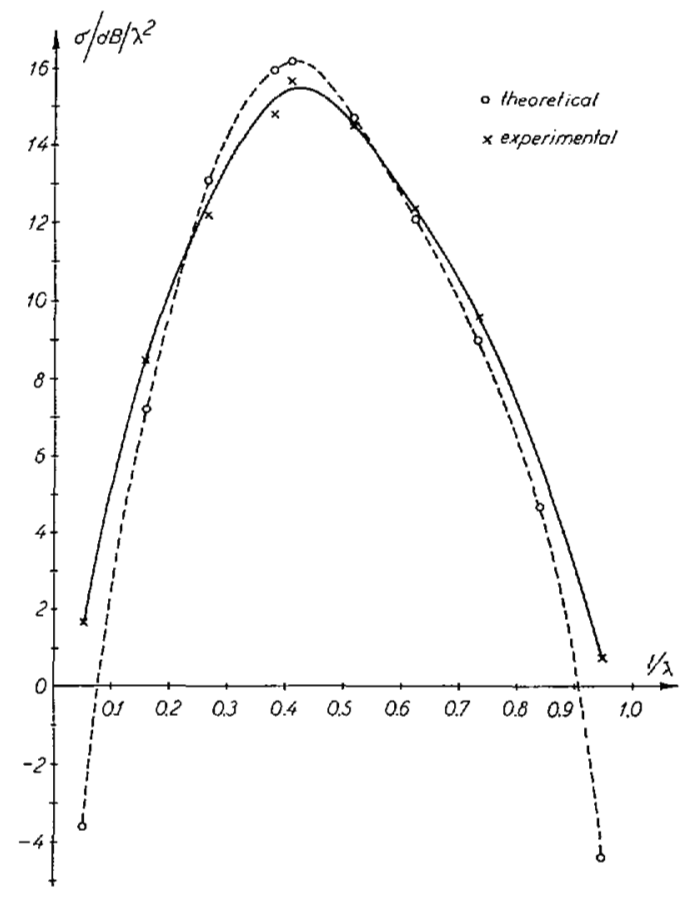

Fig. 2. Back-scattering cross section for normal incidence as a function of the length of the transmission lines. Distance butween the dipoles equals $8 \mathrm{~cm}$.

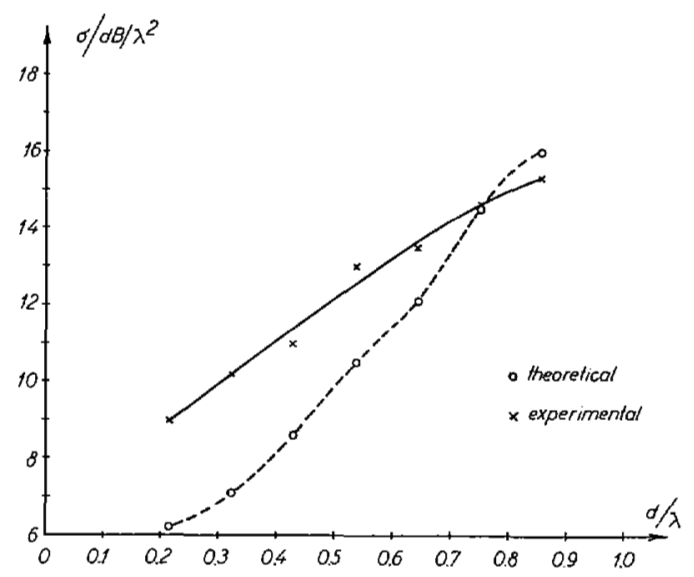

Fig. 3. Back-scattering cross section for normal incidence as a function of the distance between the dipoles. Length of the transmission lines is about $0.40 \lambda+p \lambda$. 
arranged close to each other. In this connection it is interesting to find the spacing $(d)$ between the dipoles in the case of a linear reflector if we wish to arrange them within an area $A$ and require them to possess a backscattering cross section equal to that of a flat metallic plate of area $A$. Using as the backscattering cross section of a flat plate $4 \pi A^{2} / \lambda^{2}$ it is easily found that $n \simeq 4 A / \lambda^{2}$. Since one half-wave dipole in the reflector occupies the area $\lambda / 2 \cdot d$, this means that the dipoles should be arranged with an interspacing of about half a wavelength. In fact, as will be seen below, if the spacing is smaller than half a wavelength, then coupling reduces the backscattering cross section below the value $0.80 n^{2} \lambda^{2}$ derived by neglecting coupling.

\section{EXPERIMENTAL RESULTS}

Fig. 1 shows the experimental Van Atta reflector consisting of four parallel half-wave slot-fed dipoles with open-ended terminations. Line stretchers are inserted into the transmission lines connecting the dipoles. This is done in order to determine easily the length of the transmission lines for which the reflector has a maximum back-scattering cross section for normal incidence. This cross section is measured by using a conventional monostatic radar set up in an anechoic chamber. The measurements were carried out at $3.21 \mathrm{GHz}$.

First, for an equispacing of $8 \mathrm{~cm}$ between the dipoles, the cross section is measured as a function of the length of the transmission lines. The result is shown in Fig. 2 and is in agreement with the theory. It is seen that there is a maximum of reflection when the length of the transmission lines is about $0.40 \lambda+p \lambda$, where $\lambda$ is the wavelength and $p$ is an integer. As explained in [4], it is due to coupling that the maximum does not occur for the length $0.50 \lambda+p \lambda$.

Next, with the length of the transmission lines adjusted to about $0.40 \lambda+p \lambda$, the cross section is measured when the equispacing is decreased in steps of $1 \mathrm{~cm}$ from $8 \mathrm{~cm}$ to $2 \mathrm{~cm}$ (i.e., from $0.86 \lambda$ to $0.21 \lambda$ ). The results are shown in Fig. 3. The main reasons for the discrepancies between the theoretical and experimental curves are 1) that the reflections from the transmission lines, including the line stretchers, add to or subtract from those from the Van Atta array, and 2) that the line stretchers introduce VSWRs into the transmission lines. However, in agreement with theory, it is demonstrated experimentally that when the inter-element spacing in a Van Atta reflector array is decreased, then coupling reduces the back-scattering. Furthermore, it is seen that for some spacings larger than half a wavelength, coupling may increase the backscattering cross section above the value $11.1 \mathrm{~dB} / \lambda^{2}$ found by using the formula $0.80 n^{2} \lambda^{2}$ derived above when coupling is neglected.

\section{ACKNOWLEDGMENT}

The author wishes to express his appreciation to the Radiation Laboratory, University of Michigan, Ann Arbor, for the use of their microwave anechoic chamber for the experimental study carried out during his stay at the laboratory.

J. Appel-Hansen Laboratory of Electromagnetic Theory Technical University of Denmark Lyngby,_Denmark

\section{REFERENCES}

[1] L. C. Van Atta, "Electromagnetic reffector," U. S. Patent 2908002 , Serial no. 514 040, October 1959

[2] M. H. Østfeldt, "Linear array as a passive reflector," M.Sc. thesis, Laboratory of Electromagnetic Theory, Technical University of Denmark, Lyngby, 1963 (in Danish).

[3] T. Larsen, "Reflector arrays," IEEE Trans. Antennas and Propagation, vol. AP-14, pp. 689-693, November 1966.

[4] J, Appel-Hansen, "A Van Atta refiector consisting of half-wave dipoles," IEEE Trans. Antennas and Propagation, vol. AP-14, pp. 694-700, November 1966.

[5] Y. Y. Hu, "Back-scattering cross section of a center-loaded cylindrical antenna," IRE Trans. Antermas and Propagation, vol. AP-6, pp. 140-148, January 1958.

\section{A Note on the Radiation Characteristics and Forced Surface Wave Phenomena in Triangular-Grid Circular Wareguide Phased Arrays}

Abstract-Forced surface wave resonances are shown to occur in dielectric-free 45-degree triangular-grid circular waveguide phased arrays. Similar surface wave phenomena were observed in an identical grid array of rectangular waveguides in the $H$-plane of scan. In the circular wareguide array, however, the resonance is observed in the $E$-plane and is shown to be an isolated point. This isolation is further illustrated by observing certain vector symmetries in the radiation patterns.

Considerable effort has been devoted towards developing an understanding of certain anomalous resonances [1]-[7] which occur in phased-array antennas. These phenomena are manifested by total internal reflection and consequently no radiation of power by the antenna at particular angles of scan. While the existence of these phenomena was, to a degree, expected for dielectriccovered phased arrays under certain conditions $[1],[2]$, it has been shown that they can also occur in dielectric-free brick arrays of rectangular waveguides [3], [4] and in equilateral triangular arrays of circular waveguides [7]. The brick array of rectangular waveguides and a circular array with an identical grid both exhibit forced surface wave phenomena, but in different planes of scan.

The results to be described were obtained by numerically solving the vector two-dimensional integral equation for the planar array of circular waveguides [7]:

$$
\begin{aligned}
2 \sum_{j=1}^{J} A_{j} Y_{j} \Phi_{j}= & \sum_{j=1}^{\infty} Y_{j} \mathbf{\Phi}_{j} \iint_{A} \boldsymbol{\Phi}_{j} \cdot E_{t} d a \\
& +\sum_{p=1}^{2} \sum_{(m)} \sum_{(n)} Y_{m n p} \mathbf{\Psi}_{m n p} \\
& \cdot \iint_{A}\left(\mathbf{\Psi}_{m n n_{p}}\right)^{*} \cdot E_{t} d a
\end{aligned}
$$

We solve for the unknown tangential electric field $E_{t}$ at the aperture $A$ of the circular wave-

Mabuscript received June 21, 1968 guides. The $\left\{\boldsymbol{\Phi}_{j}\right\}$ and $\left\{\boldsymbol{\Psi}_{m n p}\right\}$ are, respectively, the circular waveguide modes [7], $[8]$ and the periodic free-space modes required by Floquet's theorem ( $p=1$ denotes a TE mode, $p=2$ a TM mode). The $Y_{j}$ and $Y_{m n p}{ }^{\prime}$ are the respective modal admittances. The mode of excitation of the array is specified by the $\left\{A_{j}\right\}$ which are the amplitudes of the incident waveguide modes (properly normalized to the total input power). (In the results presented here, we choose only the two degenerate circular $\mathrm{TE}_{11}$ modes.)

The reflection coefficients $R_{j}$ of the $j$ th incident mode are readily found from the aperture field solution by

$$
\begin{aligned}
A_{j}+R_{j}=\iint_{A^{\prime}} \boldsymbol{\Phi}_{j} \cdot E_{t} d a \\
\quad(j=1, \cdots, J) .
\end{aligned}
$$

The radiated fields (which are related to the far fields of the singly-excited waveguide element in the infinite array environment [9]) are found from the aperture field by

$$
\begin{aligned}
E_{\theta}= & \frac{1}{\sqrt{1-T_{x}{ }^{2}-T_{y}{ }^{2}}} \\
& \cdot \iint_{A}\left(\Psi_{002}\right)^{*} \cdot E_{t} d a
\end{aligned}
$$

and

$$
E_{\dot{\phi}}=-\iint_{A}\left(\Psi_{001}^{*}\right)^{*} \cdot E_{t} d a,
$$

where the angles $\theta$ and $\phi$ are the usual polar coordinate angles associated with the $x, y, z$ coordinate system. $T_{x}$ and $T_{y}$ are the beampointing directional cosines with respect to the $x$ and $y$ axis (the ground plane). The transmission coefficients of the array-scattering matrix are proportional to the radiated field components

$$
T_{\theta}=\sqrt{Y_{002}^{\prime} / Y_{1}} E_{\theta}
$$

and

$$
T_{\phi}=\sqrt{Y_{001}{ }^{\prime} / Y_{1}} E_{\phi}
$$

when only a circular $T E_{11}$ mode is incident on the aperture. The unitary condition for the scattering matrix determines the proportionality constants.

In the brick array analyzed earlier [3], [4], where only a single mode was propagating in the waveguide, surface wave resonances were observed in the $\boldsymbol{H}$-plane of scan. In contradistinction, however, when an identical grid array of circular waveguides is excited as shown in Fig. 1, we note that a forced surface wave resonance is present in the $E$-plane of scan before the first grating lobe (denoted by the vertical arrows) appears. (The magnitude of the reflection is plotted versus the differential steering phases $\psi_{x}$ or $\psi_{y}$ along the $x$ or $y$ coordinates. They are proportional to $T_{x}$ and $T_{y}$ [7].) In the $H$-plane scan, no such resonance is observed although $\left|R_{x}\right|$ is very nearly one at grazing incidence (the heavy arrow). Small changes in the wavelength do not greatly alter the results except that total reflection at grazing incidence is observed in the $H$-plane at other wavelengths.

In addition, it is of interest to note that, unlike the results found for the brick array of rectangular waveguides, the surface wave resonances found here (in the $E$-plane here and for other planes in different grids [7]) occur at isolated points in the scan plane. This 